\title{
Class D Power Amplifier For Medical APPLICATION
}

\author{
Wei Cai ${ }^{1}$, Liang Huang ${ }^{2}$ and ShunQiang Wang $^{3}$ \\ ${ }^{1}$ Department of Electrical Engineering and Computer Science, University of California, \\ Irvine, CA, USA \\ ${ }^{2}$ Department of Information \& Electronic Engineering, ZheJiang Gongshang University, \\ Hang Zhou, Zhejiang, China \\ ${ }^{3}$ Department of Mechanical Engineering, Lehigh University, Bethlehem, PA, \\ USA
}

\begin{abstract}
The objective of this research was to design a $2.4 \mathrm{GHz}$ class AB Power Amplifier (PA), with 0.18um Semiconductor Manufacturing International Corporation (SMIC) CMOS technology by using Cadence software, for health care applications. The ultimate goal for such application is to minimize the trade-offs between performance and cost, and between performance and low power consumption design. This paper introduces the design of a $2.4 \mathrm{GHz}$ class D power amplifier which consists of two stage amplifiers. This power amplifier can transmit $15 \mathrm{dBm}$ output power to a $50 \Omega$ load. The power added efficiency was $50 \%$ and the total power consumption was $90.4 \mathrm{~mW}$. The performance of the power amplifier meets the specification requirements of the desired.
\end{abstract}

\section{KEYWORDS}

Two stage, Class D, Power amplifier, Healthcare

\section{INTRODUCTION}

Wireless medical sensor networks have offered significant improvements to the healthcare industry in the 21st century, technology changes our life at everywhere[1][2][3][4]. Devices are arranged on a patient's body and can be used to closely monitor the physiological condition of patients [5][6][7][8][9][10][11][12][13]. These medical sensors monitor the patient's vital body parameters, such as temperature, heart rate, blood pressure, oxygen saturation, and transmit the data to a doctor in real time [2]. When a doctor reviews the transmitted sensor readings, they can get a better understanding of a patient's health conditions. The benefit for the patients is that they do not need to frequently visit the hospital, thus patients could reap time and money savings. Such wireless medical sensors will continue to play a central role in the future of modern healthcare. People living in rural areas would especially benefit, since $9 \%$ of physicians work in rural areas while almost $20 \%$ of the US population lives there [2]. A shortage of physicians and specialist is a big issue in such areas, even today. But Wireless Medical Sensor Network technology has the potential to alleviate the problem. In a wireless sensor network, as seen in the figure 1 below, each device is capable of monitoring, sensing, and/or displaying information. A sensor node is capable of gathering sensory information, processing it in some manner, and communicating with other nodes in the network[2]. 


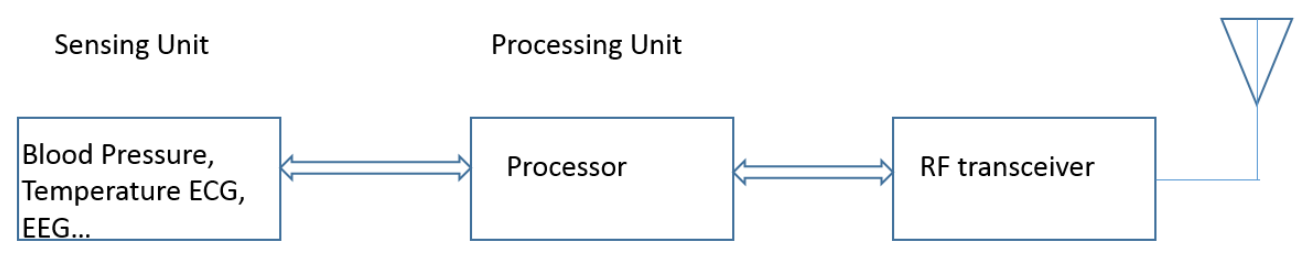

Figure 1. Block diagram of a typical sensor node

Figure 1 shows that the basic sensing node can collect the physiological signals (e.g.: such as EEG, ECG, body temperature, blood pressure, heart beat etc.), when attached to a human body [14]. The processing unit processes all the sensed signals, then sends out the data based on communication protocols. All the processed data will be transmitted through a wireless link to a portable, personal base-station. Doctors can then obtain all the patients' data through the network.

The main challenge for such sensor node is the high power consumption of portable devices. A solution to this challenge is the integration of the portable devices' digital and RF circuitry into one chip.

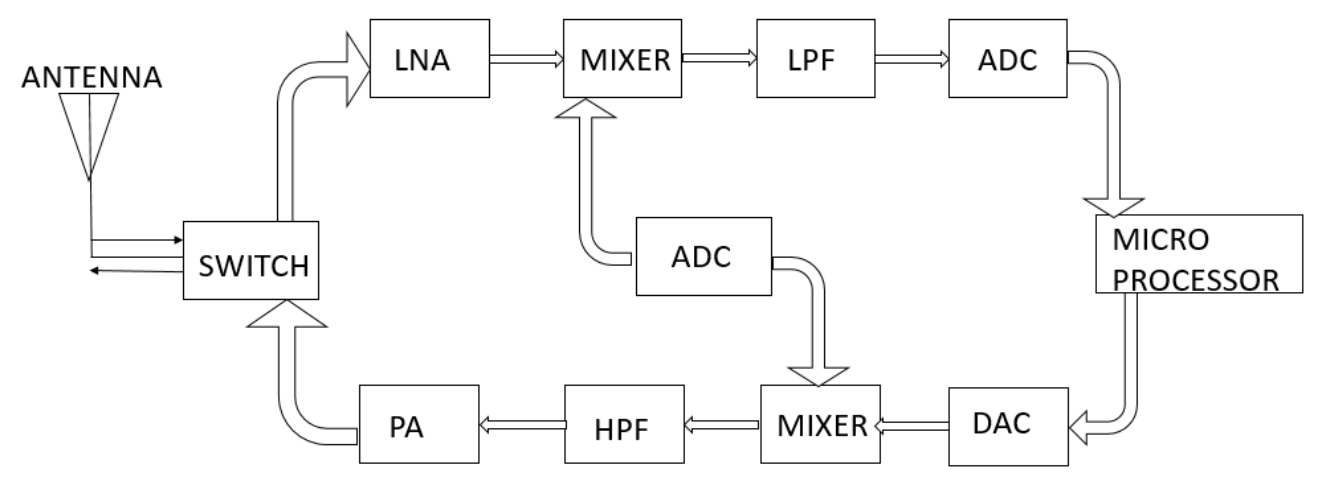

Figure 2. Block diagram of a transmitter

The receiver will receive the signal and will also perform DSP processing after the data is sent out by the transmitter [2]. Figure 2 is the transmitter diagram. It is desirable that the transmitter and receiver are low power devices. The director-conversion transmitter is very popular for such applications, because it offers versatility, flexibility, spectral efficiency, and low complexity. These features make the transmitter simpler than the super-heterodyne transmitter. Small chip and circuit size, and low power consumption can be achieved with a direct-conversion transmitter architecture. For the front-end transmitter, the major objectives are 1) transmit RF signals and 2) recover the biosignal classification. This paper proposes a low power receiver design. This paper is mainly for the power amplifier design, since other portions of the circuit design are already discussed in the paper [14]. In order to meet the standards, the PA is designed as shown in table 1. 
Table 1: PA design requirement.

\begin{tabular}{|l|l|}
\hline Parameter & Target(Unit) \\
\hline Output Power & $10 \mathrm{dBm}$ \\
\hline PAE & $40 \%$ \\
\hline Stability & $>1$ \\
\hline S11 & $-10 \mathrm{~dB}$ \\
\hline
\end{tabular}

\section{MethodS}

Over the past 30 years, research on CMOS radio-frequency (RF) front-end circuits has progressed extremely quickly. The ultimate goal for the wireless industry is to minimize the trade-offs between performance and cost, and between performance and low power consumption design [15].

The proposed Class D amplifier has low output power and good linearity based on the IEEE $802.11 \mathrm{~b}$ communication protocol. The Class-D amplifier, whose basic form is seen in figure 3, consists primarily of two switches. During device operation, the switches will alternately turn on and off due to the driver stage, and the output voltage of the switch varies between ground and the supply voltage. Also within the device is a bandpass filter composed of a series LC resonator tuned to the desired frequency, providing nonzero conductance essentially only in the band of interest. Such filters allows the maximization of the current in the frequency band of interest, while minimizing the current flowing at the unwanted band. Hence, no power dissipation happens outside of the desired operational band[16].

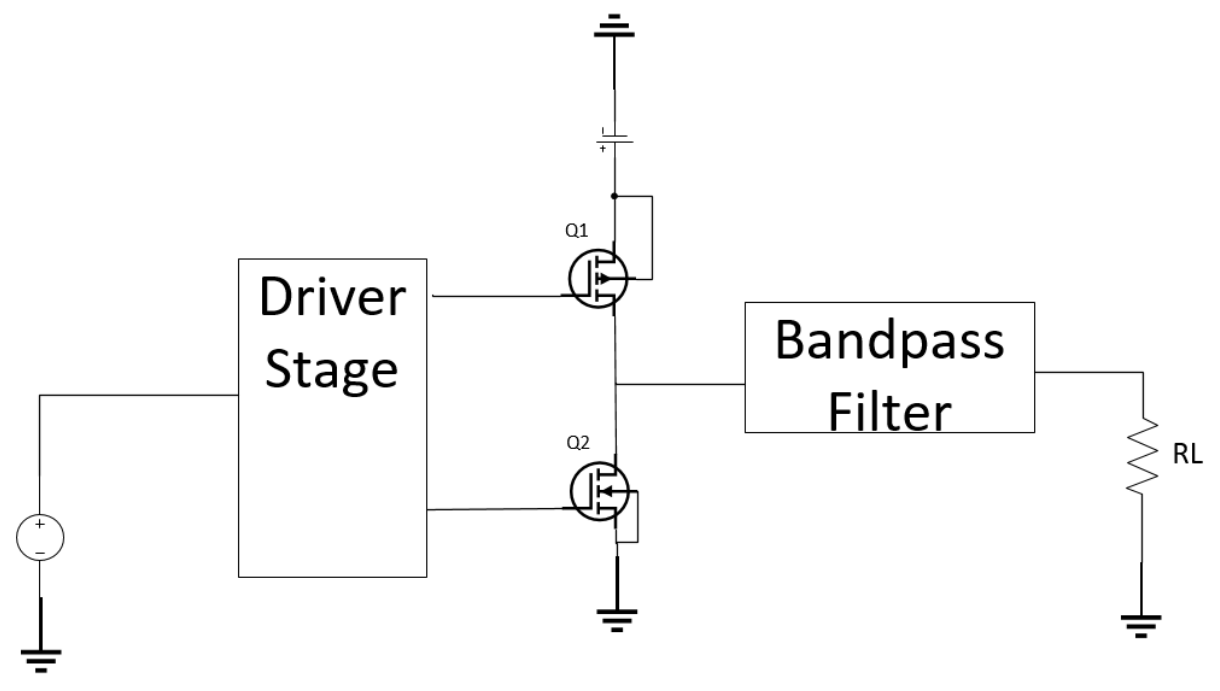

Figure 3. Block diagram of a class D power amplifier 


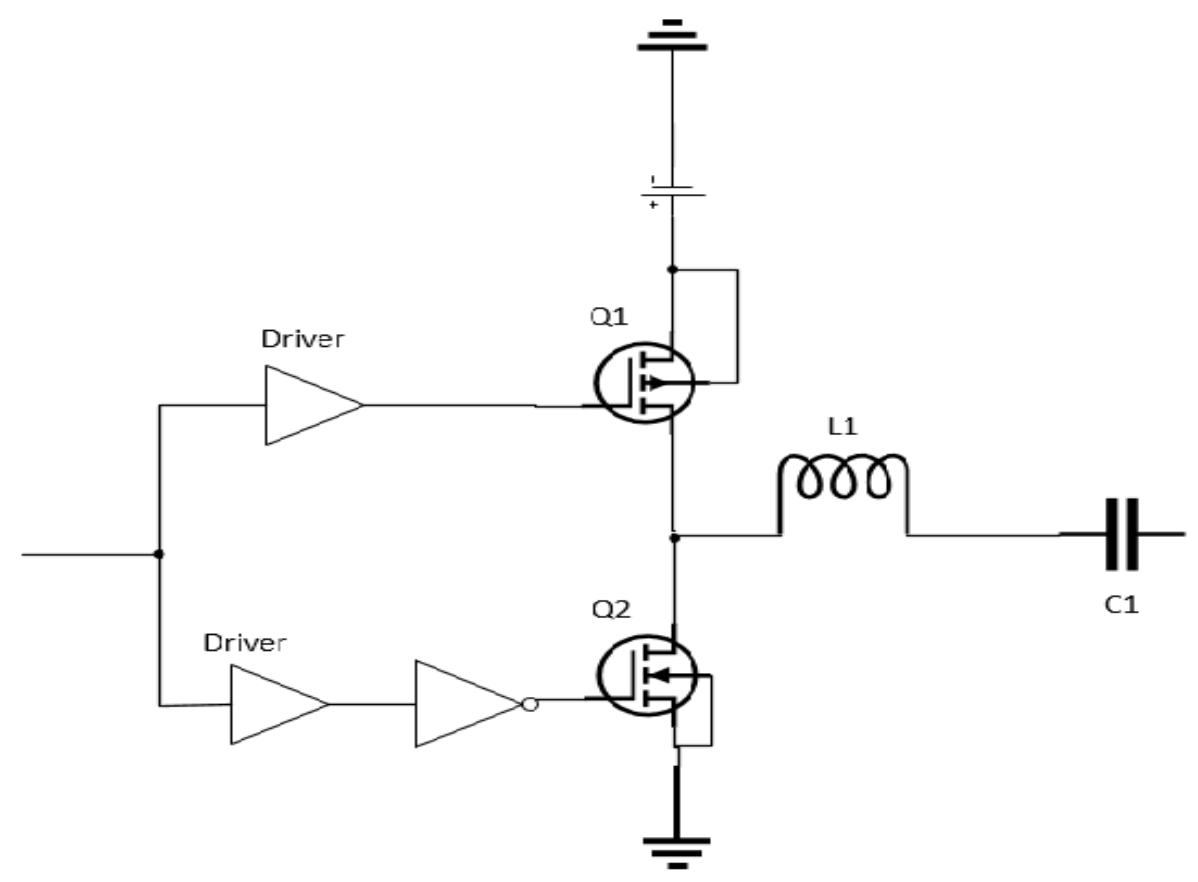

Figure 4. Schematic of PA design

To get the optimum bias, small-signal simulation and $1 \mathrm{~dB}$ compression point simulation are completed by their power output capability. Resulting design values can be shown in Table 2.

Table 2: 2.4GHz PA driver stage component.

\begin{tabular}{|l|l|}
\hline Parameter & Size (Unit) \\
\hline Q1 & W/L=1um/0.5um $(\mathrm{f}=8, \mathrm{~m}=18)$ \\
\hline $\mathrm{Q} 2$ & $\mathrm{~W} / \mathrm{L}=1 \mathrm{um} / 0.6 \mathrm{um}(\mathrm{f}=2, \mathrm{~m}=12)$ \\
\hline $\mathrm{L} 1$ & $2 \mathrm{nH}(\mathrm{Q}=20)$ \\
\hline $\mathrm{C} 1$ & $600 \mathrm{fH}$ \\
\hline
\end{tabular}

\section{RESULTS}

As seen in figure 5(a), the output power was 16 . As seen in figure $5(\mathrm{~b})$, the frequency is at $2.4 \mathrm{GHz}$ the $\mathrm{S} 11$ is less than $-10 \mathrm{~dB}$, also, the total power of the PA is $90.4 \mathrm{~mW}$.

As seen in figure 6(a), $\mathrm{Kf}$ is larger than 1 for all frequencies from 1 to $3 \mathrm{GHz}$, so this circuit was totally stable. And the PAE could reach $50 \%$. 


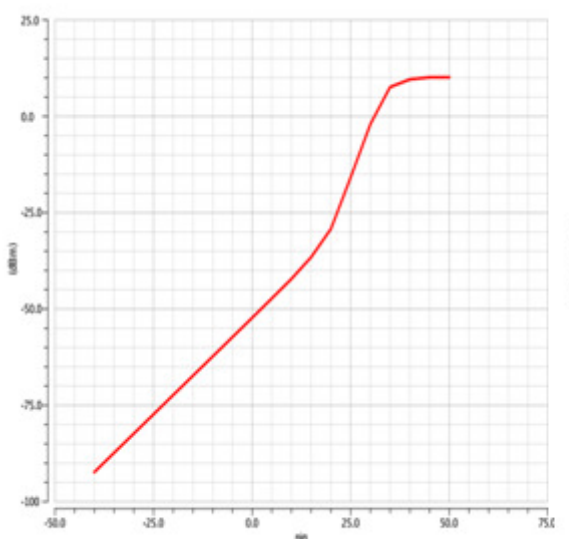

(a)

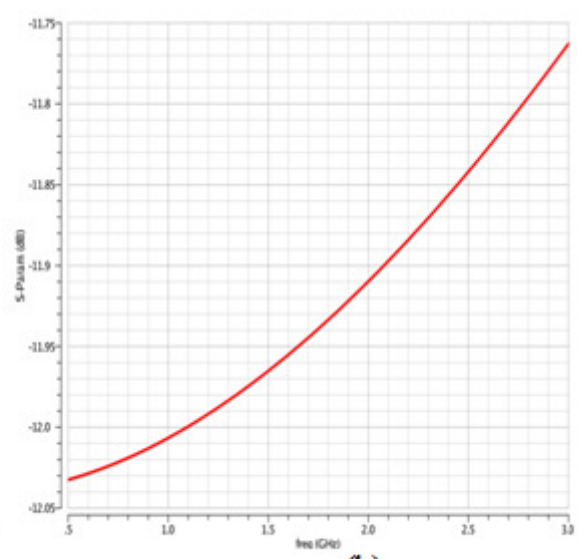

(b)

Figure 5. (a) Output power (b) S11

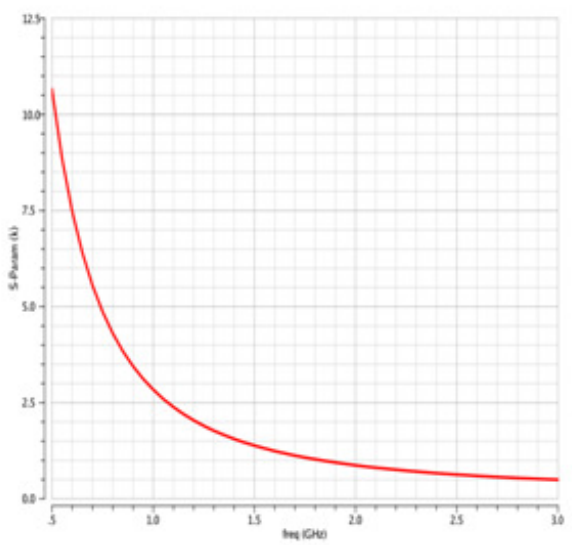

(a)

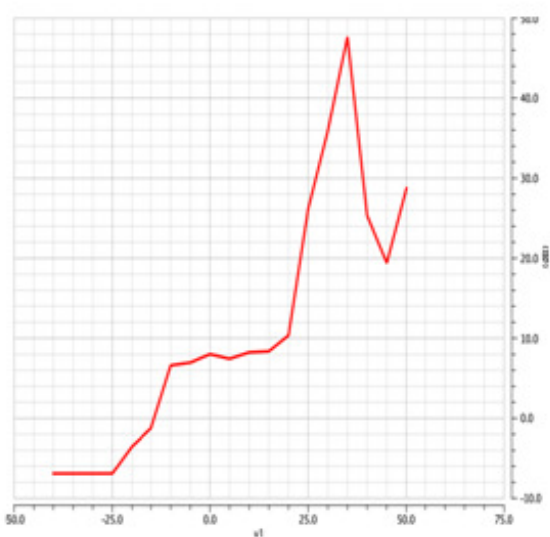

(b)

Figure 6. (a) Kf (b) PAE

\section{Conclusion}

This paper describes the method of designing and simulating power amplifier using cadence software based on SIMC CMOS process 180nm technology. This PA is used for sensor networks. This research is still in the early stages of development of a low cost and low power device. In order to reach the performance that is needed, the PA process uses group III and IV elements. This circuit meets the scheduled requirements for the CMOS process, but it still has room to improve performance metrics. When the sensor is coupled with communications technologies such as mobile phones and the Internet, the sensor network constant information flow between individuals and their doctors. Such low cost and low power device can save a lot of hospitalization resources. To realize this, future improvement is needed. 


\section{REFERENCES}

[1] Stults BM., (1984) "Preventive Health Care for the Elderly", Western Journal of Medicine, pp 832- 845.

[2] Afsaneh Minaie, Ali Sanati-Mehrizy, Paymon Sanati-Mehrizy \& Reza Sanati-Mehrizy (2013). "Application of Wireless Sensor Networks in Health Care System", ASEE, vol 3, pp 21-24.

[3] Narayanunni, Vinay, Heng $\mathrm{Gu}$, and Choongho $\mathrm{Yu}(2011)$ "Monte Carlo simulation for investigating influence of junction and nanofiber properties on electrical conductivity of segregated-network nanocomposites." Acta Materialia 59.11 pp 4548-4555.

[4] Gu, H., X-L. Gao, and X. C. Li(2009)"Molecular Dynamics Study on Mechanical Properties and Interfacial Morphology of an Aluminum Matrix Nanocomposite Reinforced by -Silicon Carbide Nanoparticles." Journal of Computational and Theoretical Nanoscience 6.1 pp61-72.

[5] Q Zhang, X Xiao, YT Cheng, MW Verbrugge (2014)"A non-destructive method for measuring the mechanical properties of ultrathin films prepared by atomic layer deposition", Applied Physics Letters 105 (6), 061901

[6] X Yu, X Wang, Q Zhang, J Li, J Liu (2014) " Oxidation-resistant, solution-processed plasmonic Ni nanochain-SiOx $(x<2)$ selective solar thermal absorbers," Journal of Applied Physics $116(7)$, 073508.

[7] H Liu, J Liu, Y Liu, K Bertoldi, CD Vecitis (2014) "Quantitative 2D electrooxidative carbon nanotube filter model: Insight into reactive sites”, Carbon 80, pp 651-664.

[8] Qinghua Wang, Huimin Xie, Jia Liu, Xue Feng, Fulong Dai(2009) “ Instability and failure analysis of film-substrate structure under electrical loading“, International Conference on Electronic Packaging Technology \& High Density Packaging, pp 1027-1029.

[9] Liang Wanga, Sajjad H. Marufa, Devid Manigliob, c, Yifu Ding (2012)“ Fabrication and characterizations of crosslinked porous polymer films with varying chemical compositions”, Polymer, Volume 53, Issue 17, pp 3749-3755.

[10] Liang Wanga, Yifu Ding (2015)"Creating micro-structured hydrogel-forming polymer films by photopolymerization in an evaporating solvent: Compositional and morphological evolutions", European Polymer Journal, Volume 66, pp 99-107.

[11] Wei Cai , Leslie Lauren Gouveia, "Modeling and simulation of Maximum power point tracker in Ptolemy”, Journal of Clean Energy Technologies, Vol. 1, No. 1, 2013 , PP 6-9.

[12] Wei Cai, Jeremy. Chan, David Garmire, “3-Axes MEMS Hall-Effect Sensor," presented by the 2011 IEEE Sensors Applications Symposium, pp141-144.

[13] Wei Cai, Xuelin Cui, Xiangrong Zhou, "Optimization of a GPU Implementation of Multidimensional RF Pulse Design Algorithm," International Conference on Bioinformatics and Biomedical Engineering 2011

[14] Wei Cai \& Frank Shi, (2016) “2.4 GHz Heterodyne Receiver for Healthcare Application”, IJPPS, vol 6,pp 1-7.

[15] Bo Shi, Michael Yan Wah Chia (2011) "On The Performance of Class-D Power Amplifiers With RF Pulse-Width Modulation", Proceedings of the Asia-Pacific Microwave Conference, pp15501553.

[16] Jianhui Cui, Ke Zhang, Tong Tian (2013)“A Dual-Level and Dual-Band Class-D CMOS Power Amplifier for IoT Applications”, IEEE 11th International New Circuits and Systems Conference. 


\section{Authors}

Wei Cai is a graduate student at the University of California, Irvine, CA. She received her Masters degree from Dept. of Electrical Engineering, University of Hawaii at Manoa and Bachelor degree from Zhejiang University, China. Her research interests include device physics simulation, analog/ RF circuit design.

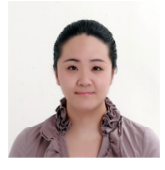

Liang huang is an associate Professor, Electronics College of Zhejiang Gongshang University. He got phd from Zhejiang University china, and finished his postdoc at Polytechnic of Turin, Italy, and Hanyang University, Seoul, Korea. His research is mainly focus on Research on: Intelligent Control; Electrical Robotics.

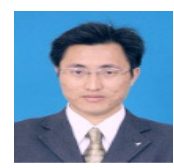

Shunqiang Wang received his Ph.D. in Mechanical Engineering from Lehigh University in 2016. His research interests are in the development of microfluidic devices and point-of-care devices for biomedical and chemical applications.

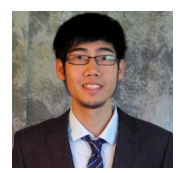

